\title{
Silicon Drift Detectors for Chemical Analysis on the nm-Scale and Below
}

\author{
M. Falke*, A. Kaeppel*, R. Terborg* \\ *Bruker Nano GmbH, Schwarzschildstraße 12, 12489, Berlin, Germany.
}

To facilitate any application in the nm-range, be that molecular cages for drug delivery in the human body or a nanostructure for semiconductor industry, it is necessary to have means for their chemical analysis. To successfully realize nm-structures it is vital to be able to control the location of each element on an even smaller scale within a reasonable amount of time. Modern energy-dispersive Xray spectroscopy (EDS) in electron microscopy is a straightforward method for chemical analysis. Today, high brightness electron sources and aberration correction in scanning transmission electron microscopy (STEM) in combination with modern liquid nitrogen free silicon drift detectors (SDD) for EDS allow the characterisation on the micrometer, $\mathrm{nm}$ and even atomic scale.

Compared to liquid nitrogen cooled EDS-detectors, SDDs [1] offer more convenience, a smaller carbon footprint and a range of new analytical possibilities for EDS in STEM. We will present EDS data achieved so far using SDD technology which demonstrate the capabilities and advantages of this young technique and show useful acquisition strategies. In S/TEM, EDS is particularly valuable for chemical analysis in case of many different elements being present at once in various quantities, like e.g. in life science. Furthermore, EDS is of interest for magnetic materials, heavier elements used in catalysis and III-V-semiconductors, where the analysis using electron energy loss spectroscopy (EELS) is either complicated due to artefacts and line overlaps or less sensitive due to the higher background in the electron spectra at higher energy losses. Fig. 1 shows, how easily and quickly small quantities of light and heavy elements can be determined. The $\mathrm{CuO}$ coating and the incorporated Co catalyst particles of multiwall carbon nanotubes can be clearly distinguished in three minutes. Recently, atom column EDS at 1.47 Angstrom column distance using Ga-K and As-K lines above $9 \mathrm{keV}$ could be shown for a III-V heterostructure [2]. For this it is vital, that the EDSdetector does not disturb the performance of the aberration corrected instrument and covers a high enough solid angle. To correctly judge the performance of an EDS system, the measurement of the output vs. input count rate for a known specimen at a given electron beam current, spot size, sample thickness and detector chip distance to the sample (solid angle) at a given take off angle has to be used. It has to be taken into account that detector efficiency (output vs input count rate) and energy resolution improve with decreasing chip size.

Figure 2a shows a quantitative deconvoluted map of an InAs/InP nanowire heterostructure using SDD-EDS at $0.12 \mathrm{sr}$ solid angle and $22^{\circ}$ take off angle. Maps of a few minutes duration or line scans allow the fast quantification of the element distribution with $\mathrm{nm}$ resolution clearly showing that As and $\mathrm{P}$ substitute each other whereas the In concentration stays at 50 at $\%$. The Au catalyst sits at the nanowire tip. Figure $2 \mathrm{~b}$ explains that without deconvolution the element distribution is not correctly determined due to line overlaps. Therefore, it is very important to use EDS systems with high energy resolution and a transparent analysis software allowing to specify overlapping lines and fully control the background determination and deconvolution procedure.

[1] Strüder L., et al., Microsc. Microanal. 4, (1999) 622-631.

[2] M.-W. Chu, et al., PRL 104 (2010) 196101. 
FIG. 1 Multiwall carbon nanotubes, $\mathrm{CuO}$ coated, with Co-catalyst particles. Map Time: 200s, solid angle $0.12 \mathrm{sr}$, take off angle $22^{\circ}$, Jeol $2200 \mathrm{FS}$.
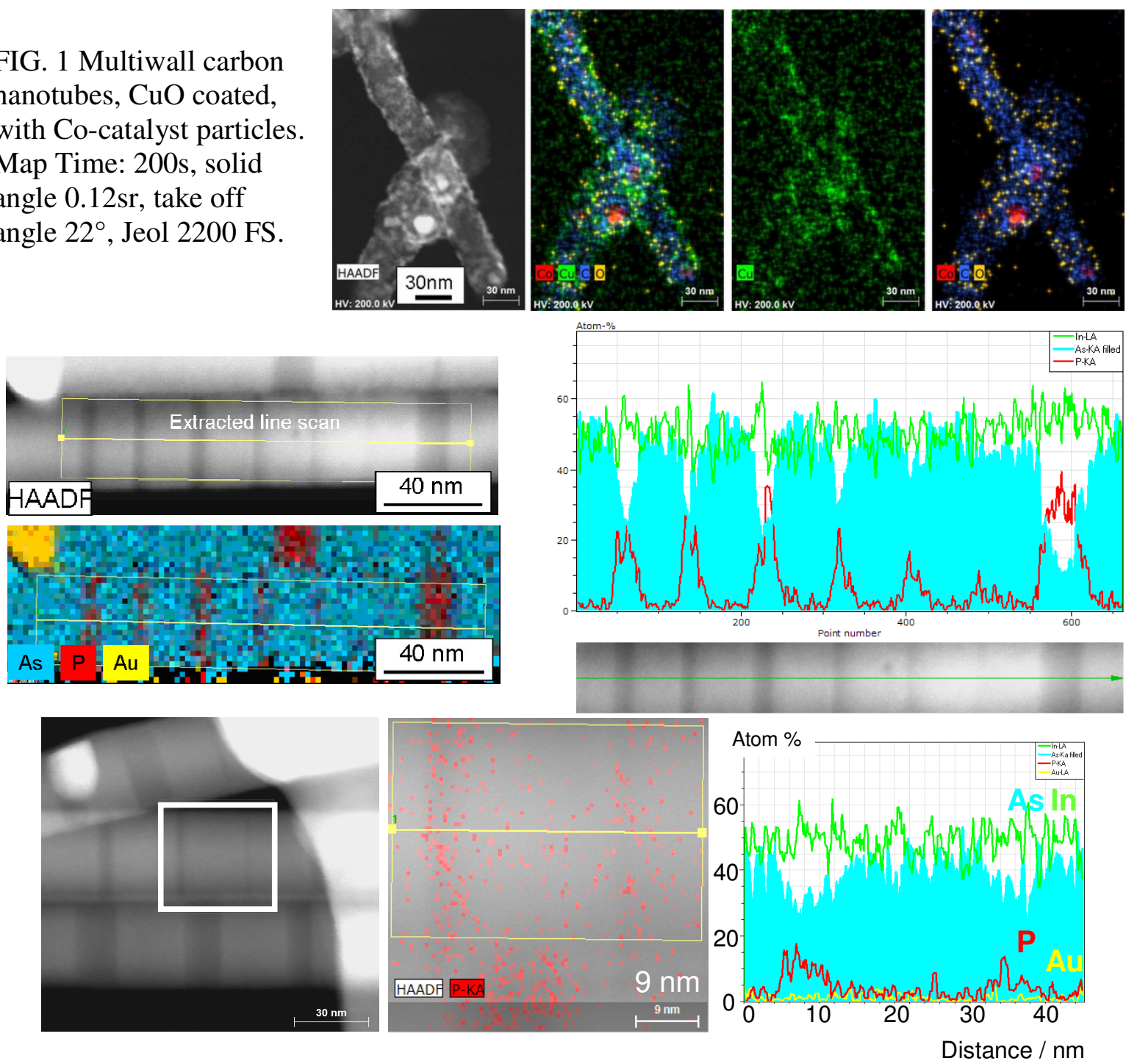

FIG. 2a Quantitative EDS map of an InAs/InP nanowire heterostructure and extracted quantitative line scans in nm resolution. Sample courtesy: D. Ercolani, L. Sorba, NEST, Pisa, Italy; mapping time: large: $18 \mathrm{~min}, 4 \mathrm{x} 4$ binning for quantification, small: $4 \mathrm{~min}$ at $400 \mathrm{pA}$ and $1 \mathrm{~nm}$ spot size.
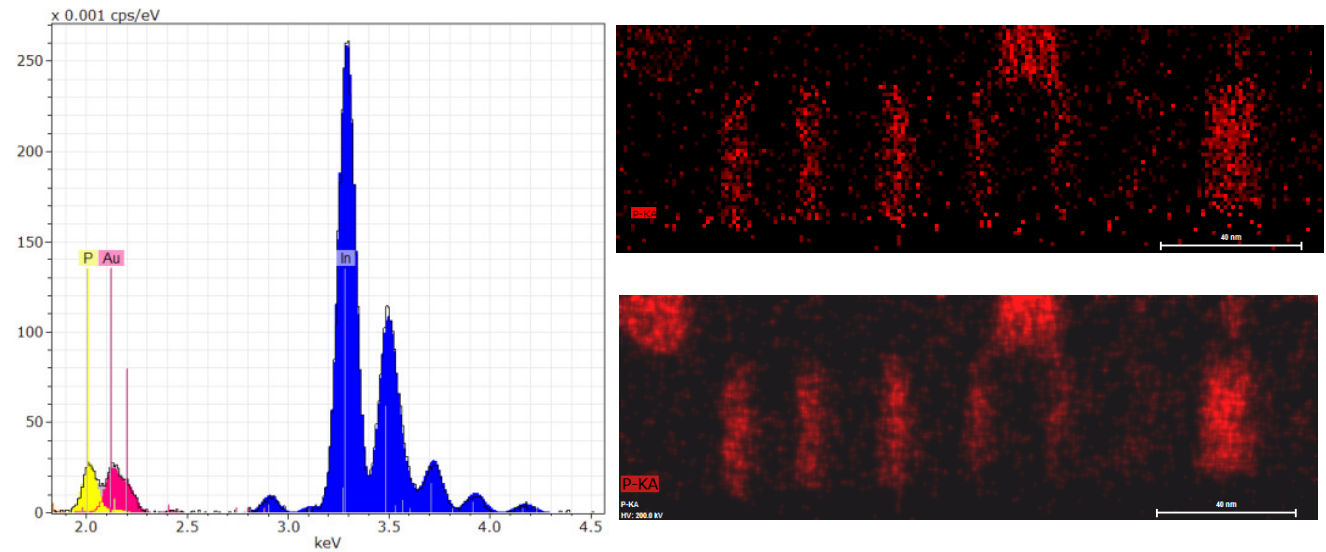

FIG. 2b left: Part of spectrum, overlap of the $\mathrm{Au}$ M-alpha with P K-alpha line. Top right: $\mathrm{P}$ distribution after deconvolution using $4 \times 4$ binning, below: without deconvolution. 